\title{
Optimisation du fonctionnement des atténuateurs de houle de type «dos de chameau » à l'aide de perforations dans la structure
}

\author{
David LAJOIE ${ }^{1}$ \\ ${ }^{1}$ ACRI-IN, 260 Route du Pin Montard, BP 234, 06904 Sophia-Antipolis \\ david.lajoie@acri-in.fr
}

\section{Résumé :}

Le fonctionnement de l'atténuateur de type «dos de chameau » est fondé sur le principe du mur d'eau fixe, qui permet de renvoyer une grande partie des houles abordant les structures semi-immergées, comme celles qui ont été conçues pour protéger le nouvel avant-port de Monaco. Mais contrairement aux digues semiimmergées «classiques», qui sont en général équipées de superstructures aménagées, le «dos de chameau » est conçu pour être une simple barrière antihoule, pouvant être largement franchie, au moins au niveau de la première bosse, mais avec un minimum d'impact visuel. On présente les résultats d'une étude numérique et expérimentale d'optimisation du fonctionnement de ce système, en introduisant des perforations dans la structure.

\begin{abstract}
:
Wave absorbers such as "camel back" are based on the fixed water wall principle, that allows the reflecting of the major part of the waves coming to semisubmerged structures, such as those that have been designed to protect the new outer harbour of Monaco. But in opposition to "classical" semi-submerged breakwaters, that are usually equipped with superstructures, "camel back" wave absorbers are designed as a simple wave barrier, that may be overtopped easily at least above the first hump, but with a minimal visual impact. Results of a numerical and experimental study are presented, based on introduction of perforations into the structure to optimise the system.
\end{abstract}

\section{Mots-clés :}

Atténuateurs de houle - Parois poreuses - Caissons Jarlan - Méthode des singularités - 


\section{$1 \quad$ Introduction}

Le principe du phénomène hydrodynamique de «mur d'eau », qui prend place sous les structures immergées ou semi-immergées lorsqu'elles sont sollicitées par la houle, permet de renvoyer en grande partie l'énergie de houle. On distingue les principes de fonctionnement suivants :

- le «mur d'eau oscillant», engendré par la présence d'une plaque horizontale immergée qui inhibe les mouvements verticaux associés à la houle. Les particules d'eau au droit de la plaque suivent un mouvement d'oscillation horizontal qui, pour certaines conditions de longueurs d'onde, s'oppose au train d'onde incident, provoquant une réflexion presque totale et donc une transmission quasi-nulle de l'énergie. Le procédé est efficace uniquement sur une plage de périodes, les houles longues mais aussi les petits clapots non déferlant au-dessus de la structure étant peu perturbés.

- le «mur d'eau fixe », reprenant le principe de la plaque immergée, mais avec une superstructure émergeante, permettant de stopper les petits clapots, tout en bénéficiant des réflexions provoquées par la mise en mouvement horizontal des particules d'eau sous la face inférieure de la structure. La mise en place d'un béquet à l'amont de la superstructure émergeante améliore fortement l'efficacité du dispositif par rapport à une simple digue parallélépipédique semi-immergée.

Ces principes ont déjà été appliqués au réel, avec l'installation de plaques immergées à Barcelone et avec l'extension du port de la Condamine, où la grande digue flottante tire profit du principe du «mur d'eau fixe » perfectionné à l'aide de béquets.

Des structures de type «dos de chameau », également fondées sur le principe du mur d'eau fixe, ont été testées avec succès en canal à houle, avec l'avantage de limiter la cote d'arase de l'ouvrage dans l'hypothèse d'une ligne de protection bien au large des aménagements accessibles.

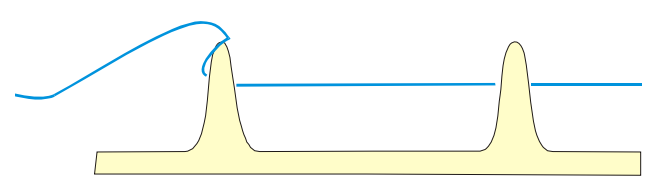

Figure 1 : schéma de principe $d u$ « dos de chameau» 
L'ouvrage n'a alors pas de superstructures aménagées, mais il est très bas audessus de l'eau, avec un faible impact visuel. Il agit alors comme une première barrière anti-houle, limitant l'ampleur de la protection directe à donner à un quelconque aménagement en mer, en particulier par grande profondeur.

De tels ouvrages, qui pourraient être montés sur jackets en grande profondeur, sont très fortement sollicités par les efforts hydrodynamiques verticaux et horizontaux. Dans le cadre des réflexions préalables au projet d'extension urbaine de Monaco, prévu dans la prochaine décennie, une réflexion a été engagée sur les possibilités de réduire ces efforts, tout en maintenant l'efficacité atténuatrice du dispositif.

L'une des pistes possibles est d'ajourer en partie la structure de l'ouvrage. Un modèle numérique bidimensionnel de diffraction avec possibilité d'introduire des parois poreuses a été développé, puis exploité pour cerner les meilleurs compromis entre efforts et efficacité hydrodynamique. Une configuration type a été retenue pour valider le principe lors d'une campagne d'essais dans le canal à houle d'ACRI.

\section{Modélisation numérique du comportement de l'atténuateur}

\subsection{Equations générales}

Le modèle numérique est fondé sur la théorie du potentiel linéarisé de la houle, avec une introduction locale des phénomènes non linéaires de perte de charge à travers les perforations. Les hypothèses ou approximations suivantes sont effectuées en dehors des zones des perforations :

- l'eau de mer est assimilée à un fluide parfait isovolume ;

- le champ de vitesses est irrotationnel en tout point du domaine fluide (D) ;

- la condition de surface libre est la condition de Poisson linéarisée des fluides parfaits, affichée sur le plan d'eau moyen (SL), de cote $\mathrm{z}=0$;

- la houle incidente est la houle d'Airy ;

- la carène $(\Sigma)$ sollicitée par la houle incidente est fixe.

Il en résulte :

- d'une part, que l'écoulement qui s'établit autour de la barge est régi par l'existence d'une fonction de potentiel harmonique $\phi(\mathrm{M}, \mathrm{t})$; 
- d'autre part, que ce potentiel est une fonction sinusoïdale du temps dont la pulsation $\omega$ est égale à celle de la houle incidente.

On posera alors $\phi(M, t)=\mathfrak{R}_{e}\left(\phi(M) e^{-i \omega t}\right)$

$\phi(\mathrm{M})$ étant l'amplitude complexe de $\phi(M, t)$.

Cette notation sera généralisée à toute grandeur dépendant linéairement de ce potentiel : la pression $\mathrm{P}$, la vitesse $\overrightarrow{\mathrm{V}}$, la déformée $\eta$ de la surface libre,...; par exemple : $p(M, t)=\mathfrak{R}_{e}\left[\mathbf{P}(M) e^{-i \omega t}\right\rfloor$

Dans ces conditions, on est ramené à résoudre un problème stationnaire caractérisé par le système d'équations linéaires suivant :

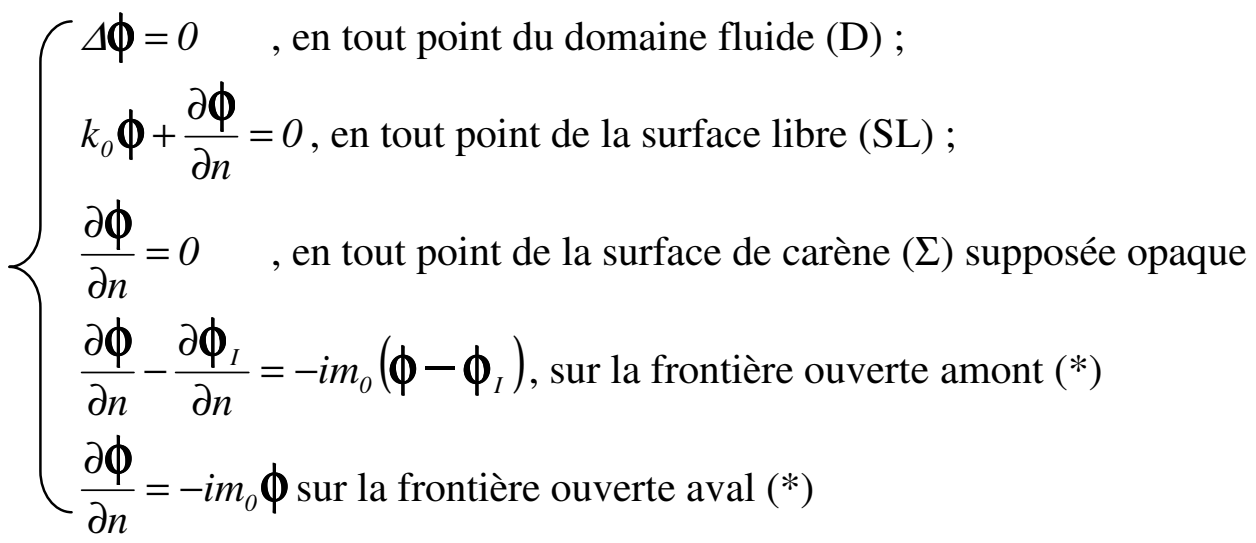

où on a noté :

$k_{o}=\omega^{2} / g$,

$m_{0}$, le nombre d'ondes au niveau des frontières ouvertes verticales,

$\vec{n}$, la demi-normale à $(\Sigma)$ qui, par convention, sera celle qui pointe vers le fluide,

$\phi_{I}$, le potentiel complexe de la houle incidente.

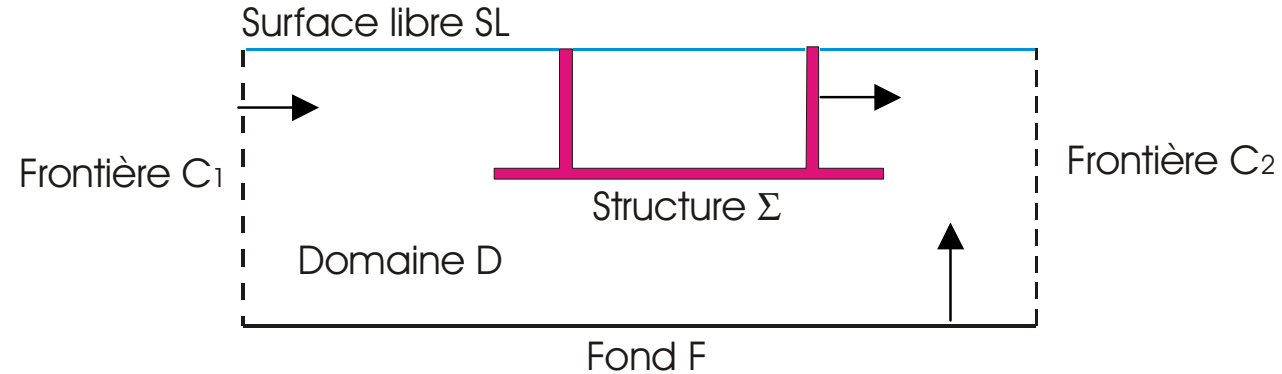

Figure 2 : Schématisation des frontières physiques du problème bidimensionnel

$(*)$ : Cette condition de non réflexion suppose que la frontière ouverte est suffisamment éloignée de la structure pour que l'onde y soit purement progressive. 
Une fois déterminée la solution de ce système, on connaît le potentiel $\phi$ sur toutes les frontières du domaine. On en déduit classiquement, par application de la formule de Lagrange linéarisée, l'expression de la pression, et donc des efforts sur la structure, ainsi que l'expression des élévations de surface libre de chaque côté de la structure.

\subsection{Résolution du problème numérique par la méthode des singularités}

On admet, moyennant les hypothèses décrites précédemment, qu'il existe une distribution superficielle de singularités cinématiquement équivalente au corps en mouvement. Ces singularités sont distribuées sur la partie immergée du corps dans sa géométrie à l'état initial. Le problème de diffraction sans vitesse d'avance en profondeur quelconque est ainsi résolu par une méthode intégrale dont les équations décrivent une distribution mixte de GREEN comportant à la fois des singularités superficielles du type sources et du type doublets normaux. Pour M appartenant à la frontière $\partial \mathrm{D}$ du domaine fermé $\mathrm{D}$, il vient ainsi :

$$
\frac{1}{2} \phi(M)+\int_{\partial D}\left(\phi\left(M^{\prime}\right) \frac{\partial}{\partial n_{M^{\prime}}} S\left(M, M^{\prime}\right)\right) d l\left(M^{\prime}\right)-\int_{\partial D}\left(\frac{\partial \phi}{\partial n}\left(M^{\prime}\right) S\left(M, M^{\prime}\right)\right) d l\left(M^{\prime}\right)=0
$$

avec $S\left(M, M^{\prime}\right)=\frac{1}{2 \pi} \ln \left|M M^{\prime}\right|$

Cette équation est résolue numériquement sous forme discrétisée en admettant que le potentiel ou sa dérivée normale sont constants par segment élémentaire i de centre $\mathrm{M}_{\mathrm{i}}$. En notant $\phi_{i}=\phi\left(M_{i}\right)$, il vient :

$$
\frac{1}{2} \boldsymbol{\phi}_{i}+\sum_{j \in \partial D} \boldsymbol{\phi}_{j} D_{i j}-\sum_{j \in \partial D} \frac{\partial \phi_{j}}{\partial n} S_{i j}=0
$$

$S_{\mathrm{ij}}$ et $\mathrm{D}_{\mathrm{ij}}$ étant respectivement les coefficients d'influence des sources et doublets du segment $\mathrm{j}$ vers le segment $\mathrm{i}$.

L'équation discrétisée écrite sur tous les $\mathrm{N}$ segments i de la frontière $\partial \mathrm{D}$ forme un système linéaire à $\mathrm{N}$ équations. La connaissance de l'une des inconnues sur chaque segment (soit $\phi_{j}$, soit $\frac{\partial \phi_{j}}{\partial n}$ comme sur le fond ou la structure $\Sigma$ ) ou bien d'une relation entre $\phi_{j}$ et $\frac{\partial \phi_{j}}{\partial n}$ (comme sur la surface libre SL ou les frontières ouvertes $\mathrm{C}_{1}$ et $\mathrm{C}_{2}$ ), rend le système collocatif, à $\mathrm{N}$ inconnues. 


\subsection{Introduction de l'effet de porosité à travers une paroi}

La paroi poreuse est modélisée par une condition aux limites appropriée traduisant la perte de charge de l'écoulement séparé par jets à travers les porosités. La différence de pression de part et d'autre de la paroi poreuse s'exprime en fonction de la vitesse au carré de l'écoulement :

$$
\Delta P=\frac{1}{2} \rho K|V| V+\int_{l} \frac{\rho}{\tau k_{C}} \frac{\partial \vec{V}}{\partial t} \cdot d \vec{l}
$$

$V$ est la vitesse moyenne en amont et en aval de la paroi, $V=\mathfrak{R} e\left\{\frac{\partial \phi}{\partial n} e^{-i \omega t}\right\}$.

$\rho$ est la masse volumique de l'eau.

$\tau$ est le taux de porosité de la paroi.

$k_{C}$ est le coefficient de restriction de section d'un jet de longueur $l$ à travers une perforation. En général $k_{C}=0.65$ pour un trou circulaire. Si $v$ est la vitesse du jet à travers un trou, il vient : $V=k_{C} \tau v$.

Le coefficient $\mathrm{K}$ de perte de charge dépend principalement de la porosité de la paroi : $K=\frac{1-\tau}{\tau^{2} k_{C}^{2}}$

Pour un écoulement oscillant de période $\omega$, il vient :

$$
\begin{aligned}
& \Delta P=P^{+}-P^{-}=\Re e\left\{i \omega \rho\left(\phi^{+}-\phi^{-}\right) e^{-i \omega t}\right\} \\
& |V| V=\left|\mathfrak{R} e\left\{\frac{\partial \phi}{\partial n} e^{-i \omega t}\right\}\right| x \Re e\left\{\frac{\partial \phi}{\partial n} e^{-i \omega t}\right\}
\end{aligned}
$$

On applique la linéarisation dite de Lorentz sur la dernière équation :

$$
\left|\mathfrak{R} e\left\{\frac{\partial \phi}{\partial n} e^{-i \omega t}\right\}\right| x \Re e\left\{\frac{\partial \phi}{\partial n} e^{-i \omega t}\right\}=\frac{8}{3 \pi}\left\|\frac{\partial \phi}{\partial n}\right\| x \Re e\left\{\frac{\partial \phi}{\partial n} e^{-i \omega t}\right\}
$$

L'équation de perte de charge relie alors le potentiel de part et d'autre de la paroi à la vitesse normale comme suit :

$$
\Re e\left\{i \omega \rho\left(\phi^{+}-\phi^{-}\right) e^{-i \omega t}\right\}=\frac{1}{2} \rho K \frac{8}{3 \pi}\left\|\frac{\partial \phi}{\partial n}\right\| x \Re e\left\{\frac{\partial \phi}{\partial n} e^{-i \omega t}\right\}+\frac{\rho l}{\tau k_{C}} \Re e\left\{-i \omega \frac{\partial \phi}{\partial n} e^{-i \omega t}\right\}
$$

Cette expression devient sous forme complexe :

$$
\boldsymbol{\phi}^{+}-\boldsymbol{\phi}^{-}=-i \frac{4 K}{3 \pi \omega}\left\|\frac{\partial \phi}{\partial n}\right\| x \frac{\partial \phi}{\partial n}-\frac{l}{\tau k_{C}} \frac{\partial \phi}{\partial n}
$$

On peut inverser cette équation pour obtenir une expression de la vitesse normale en fonction du potentiel. Il vient ainsi : 


$$
\frac{\partial \phi}{\partial n}=\frac{\boldsymbol{\phi}^{+}-\boldsymbol{\phi}^{-}}{-i \frac{4 K}{3 \pi \omega}\left\{\left(-\frac{l^{2}}{\tau^{2} k_{C}^{2}}+\left[\frac{l^{4}}{\tau^{4} k_{C}^{4}}+4\left(\frac{4 K}{3 \pi \omega}\left\|\boldsymbol{\phi}^{+}-\boldsymbol{\phi}^{-}\right\|\right)^{2}\right]^{\frac{1}{2}}\right) / 2\left(\frac{4 K}{3 \pi \omega}\right)^{2}\right\}^{\frac{1}{2}}-\frac{l}{\tau k_{C}}}
$$

Cette dernière relation peut être intégrée dans la seconde formule de GREEN. Cela introduit toutefois un terme non linéaire en $1 /\left\|\phi^{+}-\phi^{-}\right\|$dans le système matriciel. Ce terme est inconnu a priori. La difficulté est surmontée en exploitant un processus itératif de résolution dont le point de départ est le problème de la surface opaque $\frac{\partial \phi}{\partial n}=0$. Les problèmes sont ensuite résolus pas à pas en imposant la différence de potentiel obtenue au pas précédent jusqu'à ce que les solutions convergent avec des variations relatives inférieures au pourcent. Il suffit d'une dizaine d'itérations pour converger convenablement. Le principe de modélisation a été validé sur plusieurs cas analytiques et expérimentaux. On confronte cidessous nos résultats numériques à des mesures de coefficients de réflexion lors d'une campagne d'essais en canal à houle au laboratoire de l'université de Caen (ROUSSET, 2000).

La maquette étudiée était une reproduction au $1 / 25^{\text {ème }}$ d'un des caissons Jarlan constituant la digue de Dieppe. On montre par ailleurs que la prise en compte du terme d'inertie en $\int_{l} \frac{\rho}{\tau k_{C}} \frac{\partial \vec{V}}{\partial t} \cdot d \vec{l}$ influence fortement la position du point optimal de fonctionnement des caissons Jarlan.

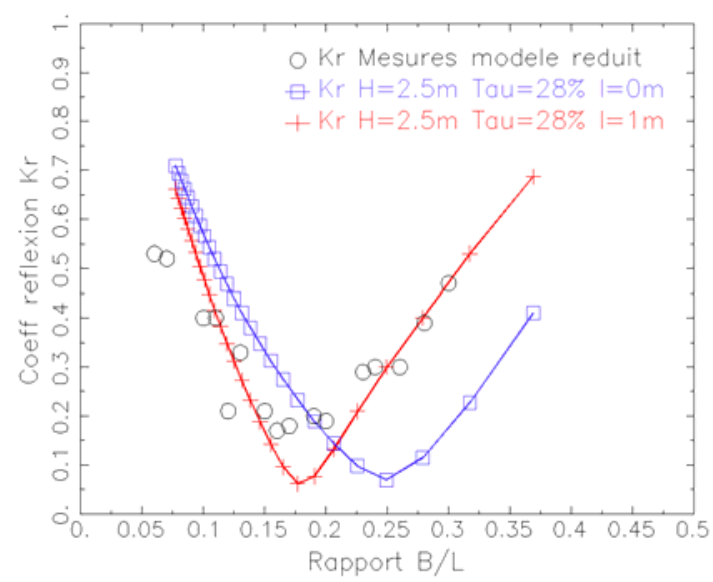

Figure 3: Coefficient de réflexion d'un caisson Jarlan de type « Dieppe »

\section{$3 \quad$ Résultats numériques}

Une étude paramétrique systématique a été menée sur de nombreuses configurations, mais sur la base de dimensions globales fixées de la structure (30m de long pour $9.5 \mathrm{~m}$ de tirant d'eau). Les graphes ci-dessous montrent l'évolution en fonction de la période du coefficient de transmission $\mathrm{K}_{\mathrm{t}}$, des efforts globaux horizontaux et verticaux $\mathrm{F}_{\mathrm{X}}$ et $\mathrm{F}_{\mathrm{Z}}$, pour plusieurs valeurs du taux de porosité de la bosse amont. 

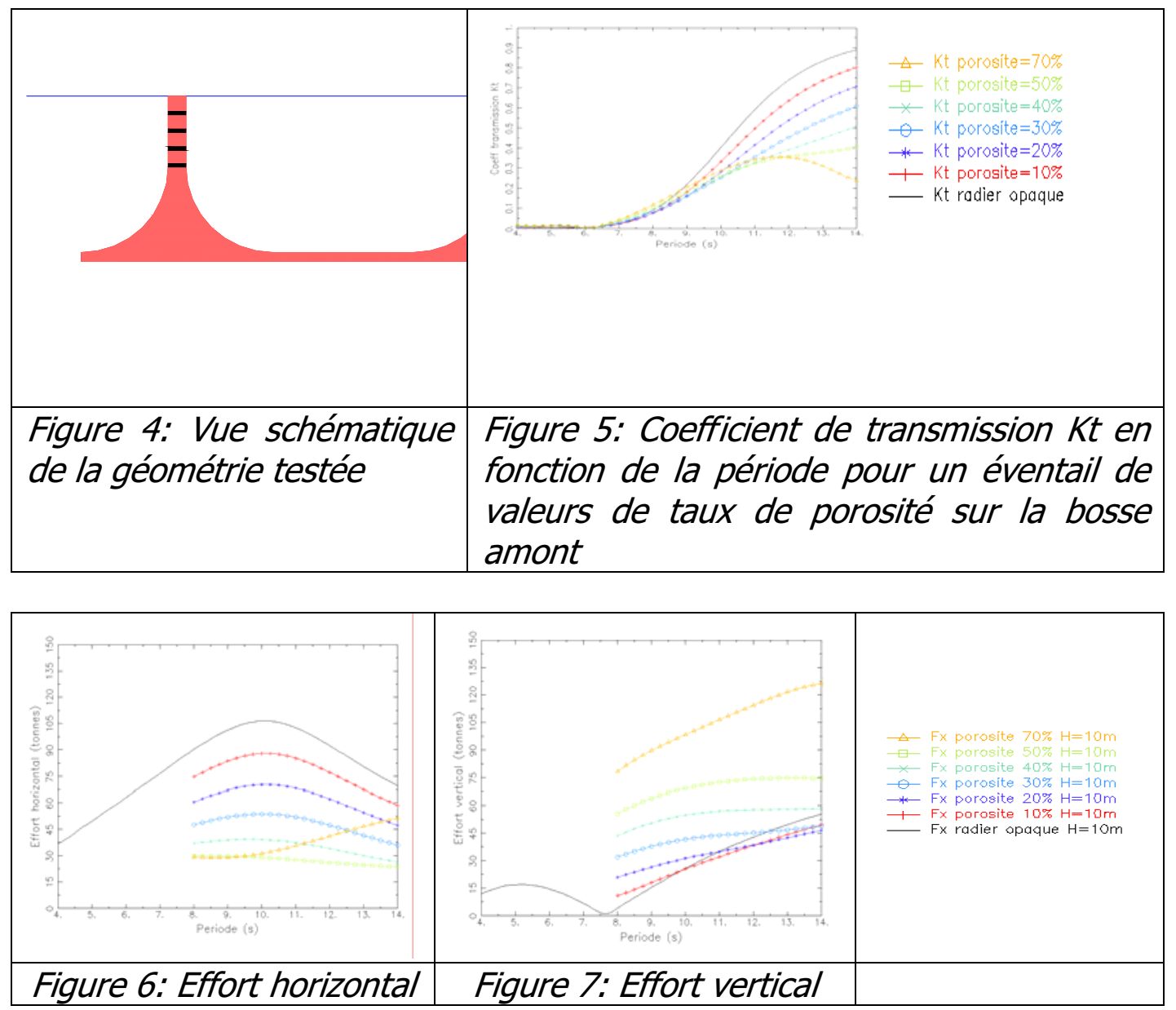

L'examen des courbes de résultats nous amène aux constats suivants :

- L'introduction d'une surface poreuse sur la bosse amont apporte une amélioration significative de l'efficacité de l'atténuateur, le coefficient $\mathrm{Kt}$ décroissant en particulier vers les plus fortes périodes.

- L'effort horizontal est aussi réduit de façon significative. Pour une structure opaque, l'effort est de l'ordre de $2.2 \mathrm{t} / \mathrm{m}^{2}$ par mètre d'amplitude « $\mathrm{a}$ » de houle ( $F x \approx 105 \mathrm{t} / \mathrm{ml}$ pour $\mathrm{H}=10 \mathrm{~m}$ ou $\mathrm{a}=5 \mathrm{~m})$, alors qu'il serait de l'ordre de $1.1 \mathrm{t} / \mathrm{m}^{2}(\mathrm{Fx} \approx 55 \mathrm{t} / \mathrm{ml})$ pour $\tau=30 \%$.

- L'effort vertical, en revanche, s'accroît fortement avec les taux de porosité élevés. En réalité, rendre la bosse amont pratiquement transparente équivaut à ramener la structure fortement asymétrique et opaque, avec une énorme plaque horizontale et une seule bosse à l'aval. On perd les effets de dissipation sur la bosse amont, mais on gagne sur le fonctionnement ondulatoire avec l'effet «plaque horizontale». Celui-ci s'accompagne toutefois de gros efforts 
verticaux et de renversement. II annihile par ailleurs l'intérêt du dos de chameau arasé bas, puisque seule la seconde bosse permet de limiter les franchissements. En définitive, un taux de porosité de l'ordre de $30 \%$ sur la bosse amont apparaît comme un bon compromis de fonctionnement de l'atténuateur. 


\section{Campagne d'essais en canal à houle}

Une campagne d'essais dans le canal à houle d'ACRI à Sophia Antipolis a permis de valider le principe de perfectionnement du fonctionnement des atténuateurs de type « dos de chameau ».

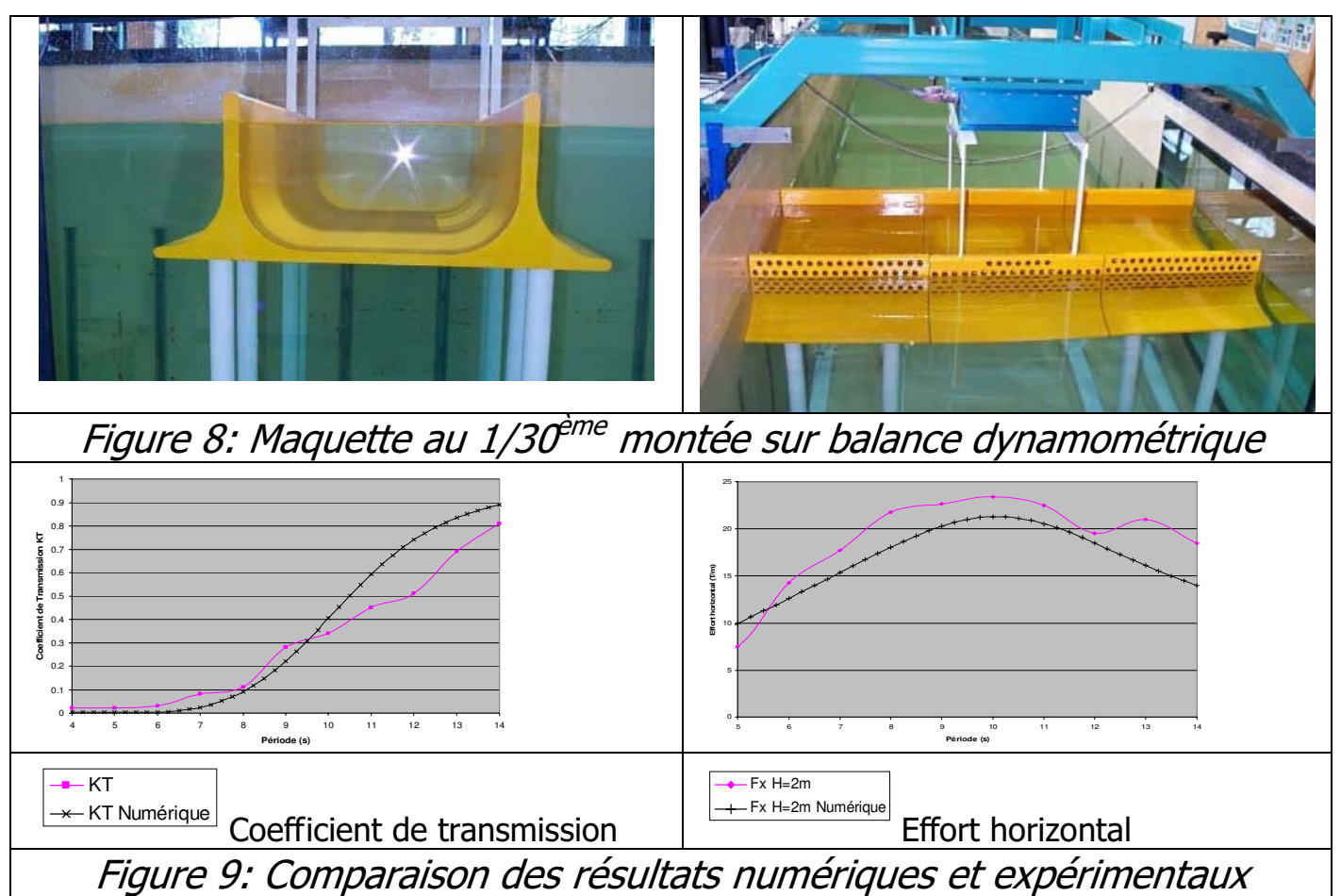

Les enseignements principaux suivants on pu être tirés de la campagne d'essais :

- Une bonne corrélation entre les calculs et les mesures, du moins pour les houles de hauteur modérée $(\mathrm{H}=2 \mathrm{~m})$, sachant que les effets de franchissement ou de dissipation externe éloignent quelque peu les résultats de calcul de la réalité physique.

- La confirmation du gain en performance de l'atténuateur de houle de type dos de chameau grâce aux perforations de la bosse amont. Les efforts horizontaux passent d'environ $1.6 \mathrm{t} / \mathrm{m}^{2}$ par mètre d'amplitude de houle à $1 \mathrm{t} / \mathrm{m}^{2}$, tandis que le coefficient de transmission $\mathrm{K}_{\mathrm{t}}$ des grosses vagues atteint 0.4 aux fortes périodes au lieu de 0.6 pour la structure opaque.

- L'intérêt modéré de perforer le radier de la structure, la baisse de performance se faisant ressentir dès les faibles taux de porosité. 


\section{$5 \quad$ Conclusion- Remerciements}

Nous avons développé un code de calcul très souple de mise en œuvre, permettant d'évaluer le comportement de structures immergées constituées de parois poreuses. L'approche entièrement numérique, fondée sur la méthode des singularités, permet d'étudier des structures perforées avec des formes quelconques, ce qui est un avantage par rapport à certains modèles de caissons fondés sur des approches semi-analytiques, souvent limités à des formes rectangulaires. L'écriture du problème uniquement aux frontières facilite aussi la création et la modification de maillages.

Le modèle numérique, exploité dans le cadre d'une recherche de perfectionnement du fonctionnement d'atténuateurs de type dos de chameau, a permis de cerner l'intérêt de perforer la structure pour à la fois réduire les efforts à reprendre et les coefficients de transmission. Le principe de perfectionnement, validé lors d'une campagne d'essais en canal à houle, a fait l'objet d'une modification du brevet international détenu par la Principauté de Monaco.

Nous remercions sincèrement le Département des Travaux Publics de Monaco, en particulier M. Manzone, pour la confiance qu'il nous a accordée dans le cadre des études prospectives de la Principauté.

\section{$6 \quad$ Références bibliographiques}

1 COLMARD C., BELLORGEY M. (1996), Effets tourbillonnaires du système mur d'eau fixe, IV ${ }^{\text {èmes }}$ journées Génie Civile - Génie Côtier, Dinard France.

2 E GUEVEL P., LANDEL E., BOUCHET R. MANZONE J.M. (1985), le phénomène du mur d'eau oscillant et son application pour protéger un site côtier soumis à l'action de la houle, ATMA.

3 MOLIN B. BETOUS P. (1993), Atténuation de la houle par une dalle horizontale immergée et perforée », $\mathrm{IV}^{\text {èmes }}$ journées de l'hydrodynamique, Nantes.

4 ROUSSET J.M, KOHL T, BELORGEY M (2000), Phénomènes dissipatifs dans une digue à paroi perforée ». VI ${ }^{\mathrm{èmes}}$ journées Génie civil Génie Côtier, Caen. 
Ouvrages portuaires et gestion des sédiments 